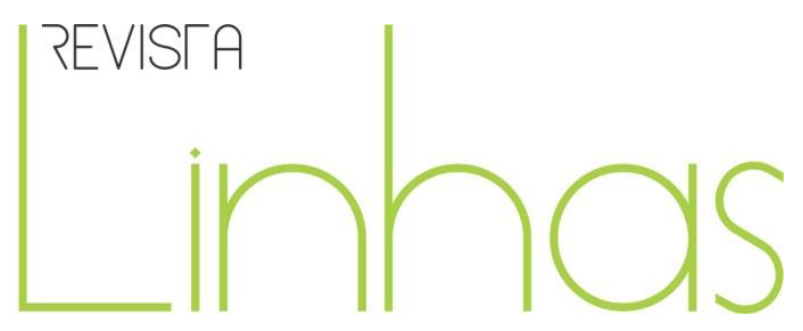

\title{
Colaboración y TIC en América Latina: el caso Interconectados
}

\section{Resumen}

La fundación y el blog Interconectados nacen en 2011 para contribuir con la generación, recopilación y movilización de conocimientos entre la universidad pública y la sociedad latinoamericana. Apoyados en la promesa de Internet y la web 2.0 de facilitar la información y la comunicación, nos hemos abocado al dictado de cursos abiertos masivos en línea (CAMEL, del acrónimo en inglés MOOC; más de siete hasta el presente), a la reflexión sobre los problemas culturales que dificultan la colaboración en nuestro medio (más de 220 entradas en el blog) y la organización de foros sobre movilización de conocimiento en alianza estratégica con la Asociación Venezolana para el Avance de la Ciencia (AsoVAC; dos realizados, uno en preparación). Nuestro objetivo general, con base en las tecnologías disponibles, es el desarrollo de capital humano y social que, conjuntamente con las leyes, normas y reglamentos que se puedan desarrollar, faciliten la integración de conocimiento útil para la toma de decisiones e insertar este conocimiento en los procesos de definición de políticas públicas para así contribuir a la solución de los problemas que aquejan a nuestra sociedad.

Palabras clave: Investigación colaborativa; Gestión universitaria; Movilización de conocimientos; CAMEL.

\section{Luis Arnoldo Ordóñez Vela}

Professor na Universidade Simón Bolívar - Venezuela. Doutor em

Bioquímica pelo Instituto Tecnológico de Massachusetts (MIT) - USA

fundacioninterconectados@gmail. com

\section{Para citar este artigo:}

ORDÓÑEZ VELA, Luis Arnoldo. Colaboración y TIC en América Latina: el caso Interconectados. Revista Linhas. Florianópolis, v. 17, n. 33, p. 82-110, jan./abr. 2016. 


\section{Collaboration and ICT's in Latin America: the Interconectados case}

\begin{abstract}
The Foundation and the blog Interconectados were born in 2011 to help in the generation, collection, and mobilization of knowledge among public universities and the Latin American society. Supported by the promise of internet and web 2.0 to facilitate information and communication, we've concentrated in massive open courses online (MOOC; CAMEL in the spanish acronym, more than seven so far), in the analysis of the cultural problems that hinder the collaboration in our part of the world (more than 220 entries on the blog) and organization of forums on mobilization of knowledge in strategic alliance with the Venezuelan Association for the Advancement of Science (AsoVAC; two made, one in preparation). Our general objective is to contribute, on the basis of the available technologies, to the development of human and social capital which, together with the laws, rules and regulations that may be develop, facilitate integrating useful knowledge for decision making and inserting this knowledge in the processes of definition of public policies based on knowledge and contribute to the solution of the problems facing our society.
\end{abstract}

Keywords: Collaborative research; University management; Knowledge mobilization; MOOC.

\section{Colaboração e TIC na América Latina: o caso do blog Interconectados}

\begin{abstract}
Resumo
A fundação e o blog Interconectados nascem em 2011 para contribuir com a geração, compilação e mobilização de conhecimentos entre a universidade pública e a sociedade latino-americana. Apoiados na promessa da Internet e da web 2.0 de facilitar a informação e a comunicação, abordamos o imperativo de cursos abertos massivos online (CAMEL, do acrônimo inglês MOOC; mais de sete até o presente), a reflexão sobre os problemas culturais que dificultam a colaboração em nosso meio (mais de 220 entradas no blog) e a organização de fóruns sobre mobilização de conhecimento em aliança estratégica com a Asociación Venezolana para el Avance de la Ciencia (AsoVAC; dois realizados, um em preparação). Nosso objetivo geral, com base nas tecnologias disponíveis, é o desenvolvimento de capital humano e social, que, conjuntamente com as leis, normas e regulamentos que se podem desenvolver, facilitem a integração de conhecimento útil para a tomada de decisões e inserir esse conhecimento nos processos de definição de políticas públicas para assim contribuir para a solução dos problemas que perturbam a nossa sociedade.
\end{abstract}

Palavras-chave: Pesquisa colaborativa; Gestão universitária; Mobilização de conhecimentos; CAMEL. 
En ocasión de presentar una ponencia en la conferencia CLACSO- Venezuela en el año 2013 en la sesión titulada La promesa de Internet: Oportunidades colaborativas en los estudios sociales en América Latina escribimos:

La percepción que de la universidad tiene el ciudadano latinoamericano promedio, pudiese ser comparable a las visiones que se tienen sobre la conquista española de este continente, se mezclan entre una leyenda negra y una leyenda dorada. La visión negra de la universidad nos habla de que Latinoamérica no tiene buenas universidades y que las mismas deben renovarse. La visión dorada, en el mejor de los casos, nos recuerda permanentemente la lógica del jarrón chino: todos la admiran, pero nadie sabe qué hacer con ella.

Creemos que en el fondo el problema reside en la generación, el levantamiento y el traslado de conocimientos desde la universidad hacia la sociedad. En nuestro medio autoritario y tradicionalista cuesta reconocer que no se sabe de algo (y si se acepta, se parte de la base que la materia no corresponde a "temas importantes") lo que dificulta el proceso de reconocer problemas, buscar información sobre los mismos, intentar hipótesis explicativas que permitan adelantar propuestas de solución y... aceptar los errores que se detecten en el posterior seguimiento y evaluación de las intervenciones, de manera de tratar de corregirlos en aproximaciones posteriores, en ese perenne buscar mejores soluciones para los problemas que nos aquejan a todos los niveles, pero fundamentalmente en el nivel social.

Lo anterior nos persigue a nivel personal desde que en el año 1978 iniciamos una campaña para que se crease en el parlamento venezolano una Comisión de Ciencia y Tecnología con el propósito de ayudar a movilizar ese deseado conocimiento en el seno del organismo llamado a supervisar y generar las políticas públicas del Estado venezolano (Ordóñez, 2015a). Muchos años han pasado, y poco ha ocurrido en nuestras sociedades desde entonces. Todavía en el 2006 el académico chileno Eduardo Devés Valdés nos recordaba: Las tareas de intelectuales y universitarios ante el estancamiento de América Latina hacia las redes y la internacional del conocimiento. Porque el problema es casi siempre visto aún como un problema personal, cuando se le pide al académico, al 
investigador, al docente universitario que "contribuya a resolver los problemas de la sociedad" sin tener en cuenta que este no puede ser un esfuerzo aislado, que por su complejidad debe ser intentando colaborativamente. La visión tradicional asume un proceso lineal que se inicia desde la sociedad que, en perfecta armonía con la universidad, logra que algún investigador genere el conocimiento que será utilizado por esa sociedad para resolver el problema originalmente planteado.

Figura 1 - La visión lineal del problema

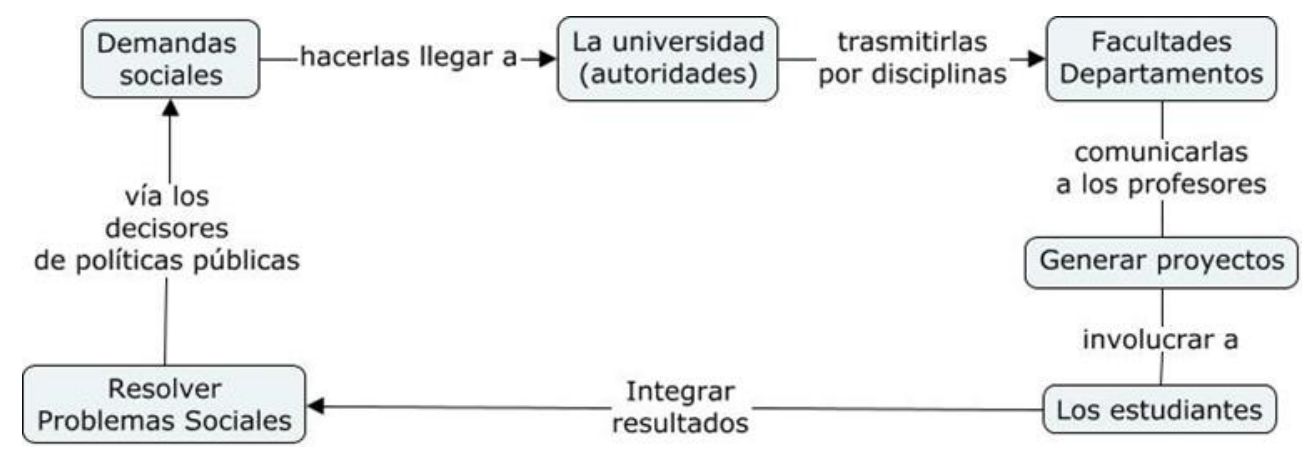

Elaboración propia.

Cuando en realidad, el verdadero problema a resolver, no es otro que el lograr que una organización débilmente acoplada, cual es la estructura universitaria, colabore a su interior y con otras instancias para integrar los conocimientos requeridos para intentar abordar los problemas complejos que presenta esa sociedad.

Figura 2 - El verdadero problema a resolver

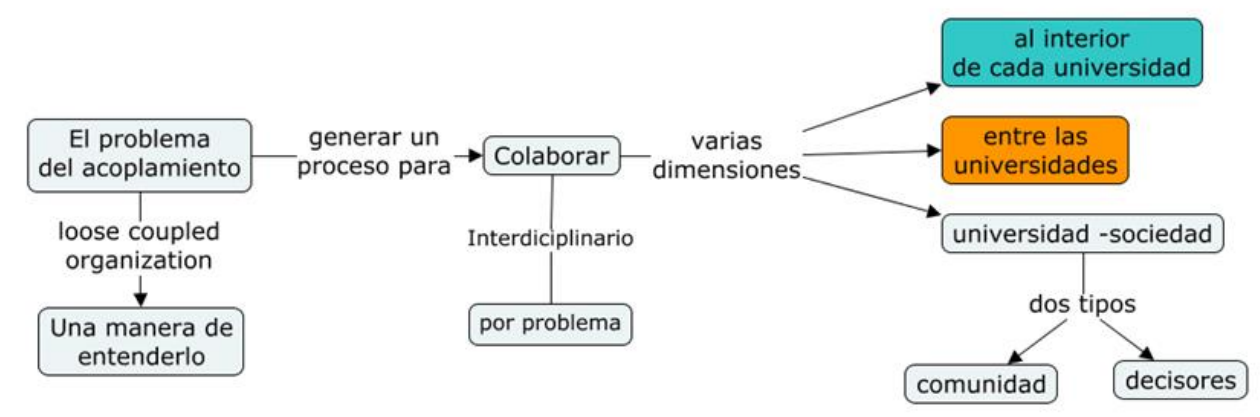

Elaboración propia 
En adición a la dificultad implícita en el proceso recién descrito se presentan problemas adicionales cuando tomamos en cuenta lo cultural, tanto al interior de la propia universidad (recordar el "Habitus" de Bordieu, 1987) como los diferentes valores que diferencian de una forma u otra a diferentes espacios culturales en el mundo (ver por ejemplo las excelentes gráficas producto de los estudios del World Values Survey, 2015).

En este último sentido, y dirigiendo la mirada a nuestra región geográfica, vale la pena tomar en cuenta los estudios de Sancho et al., 2006, quienes nos recuerdan que en los países de América Latina: “Los trabajos en colaboración representan el 65\% del total de los publicados y aumentan cada año, correspondiendo la mayor proporción a la colaboración internacional, mientras que la regional es muy escasa. Los países más productivos en ciencia presentan una colaboración relativamente menor que los pequeños productores“ (negrillas nuestras). Por su parte Aguillo et al (2005) al analizar el posicionamiento web en el sector académico iberoamericano encuentra a España más que duplicando al mejor posicionado de los países latinoamericanos para el momento del estudio (México), que, junto con Argentina, más que duplican a cualquier otro país de la región. La Unesco también nos indica (Cetto y Vessuri, 2005), que la colaboración en las universidades latinoamericanas, cuando se da, ocurre mayoritariamente con laboratorios localizados en países desarrollados bien en USA o en la Unión Europea.

Para nadie es una sorpresa que el mundo latinoamericano sufre en forma endémica de una serie de problemas sociales. Desde la pobreza, pasando por el autoritarismo, hasta la dificultad para formar ciudadanos, son toda una serie de situaciones que requieren cada vez más de investigación si es que pretendemos generar suficiente información que pueda ser utilizada a la hora de definir políticas públicas para resolverlas. Pero por su misma naturaleza de problemas complejos solo pueden ser analizados en forma compleja; es aquí donde entra a jugar un papel importante la estrategia de adelantar investigaciones colaborativas.

Resolver la situación de pobreza, por ejemplo, que es una constante en la vida de millones de latinoamericanos no es solo un problema de cultura, ni será resuelto solamente con educación, ni alterar alguna de las variables, como pudieran ser los sistemas de justicia, tendrá algún impacto, si a la vez no se resuelven las múltiples situaciones históricas que llevan a los pobres a soportar su situación. Pero acopiar y 
procesar la información necesaria para poder apenas imaginar la generación de modelos que permitan aproximaciones racionales a su solución no es tarea que pueda ser emprendida por una sola institución, menos aún por un equipo aislado de investigación.

El analizar situaciones en forma simultánea para estudiar cómo diferentes variables interactúan para generar situaciones problemáticas es una labor ciclópea, por costosa y compleja, que solo puede ser intentada cuando diferentes equipos de investigadores, con acceso a diferentes medios ambientes, comparten protocolos y suman sus inteligencias para tratar de profundizar en esos conocimientos necesarios para la toma de decisiones.

Uno de los problemas que los sociólogos todavía tienen que estudiar en nuestro medio es el porqué de nuestra dificultad a la hora de interactuar colaborativamente (de forma horizontal, no vertical autoritaria), para generar equipos eficientes en capacidad de emprender tareas complejas y llevarlas a término. Y esa es precisamente una de las trabas que tenemos a la hora de generar investigaciones colaborativas entre investigadores ubicados en diferentes países, diferentes laboratorios, diferentes disciplinas, pero alineados todos por ese espíritu que nos lleve a compartir ideas, protocolos, resultados, sueños en una suma espléndida que nos permita asesorar a los decisores desde la solidez que brinda el conocimiento generado por el método científico.

Surge entonces internet, la web 2.0 y la educación mediante los Cursos Abiertos Masivos En Línea (CAMEL) y he aquí que de repente nos encontramos ante un posible nuevo paradigma, que pudiésemos resumir en la expresión "La promesa de Internet para la universidad latinoamericana”. Un ejemplo de esta visión lo puede representar el libro editado por Web Foundation titulado Acelerando el desarrollo utilizando la WEB: Empoderando a las poblaciones pobres y marginalizados (Sadowsky, s/f). Volviendo a citar de nuestra ponencia en CLACSO:

Un camino posible para tratar de contribuir a mejorar esta situación pudiese ser el de intentar el desarrollo de capacidades en conocimiento conectivo y el surgimiento de culturas colaborativas... en el continente, de manera que mejorase la capacidad de generar, detectar y movilizar conocimiento hacia la sociedad desde la capacidad 
intelectual existente en nuestras universidades... de manera de propiciar la generación de una cultura colaborativa que nos facilitase resolver los "cuellos de botella" que en la actualidad dificultan esta movilización de conocimientos desde la universidad hacia la sociedad. Algunos de estos cuellos de botella se encuentran en las siguientes áreas (no exhaustivas, Ordóñez, 2012): aspectos culturales de la transferencia tecnológica, la transición de culturas autoritarias a culturas colaborativas y la gerencia universitaria en la sociedad del conocimiento.

En lo que sigue trataremos de esbozar el esfuerzo que hemos realizado desde la Fundación Interconectados, y recogido fundamentalmente en el blog InterConectados desde el año 2011 para tratar de paliar la situación existente.

\section{El caso InterConectados: la Fundación y el blog}

Corre el año 2011 y como parte de nuestra actividades docentes en la Universidad Simón Bolívar en Caracas, Venezuela, debemos dictar una materia electiva a nivel de pregrado cuyo proyecto nos acababa de ser aprobado. La materia en cuestión llevaba por nombre Impacto Social de Internet (CCY-655) con los siguientes objetivos:

\section{Objetivo General}

Desarrollar en los participantes capacidades analíticas sobre la definición de problemas en las ciencias sociales, utilizando para ello el tema general del impacto de las tecnologías de información y conocimiento (TIC'S) en la sociedad.

Objetivos específicos

Al finalizar el curso se aspira que los estudiantes sean capaces de:

a. Desarrollar capacidades para explorar la literatura académica universal reciente relativa al impacto social de las Tecnologías de Información y Conocimiento (TIC) en el campo educativo y en el de la participación ciudadana utilizando la web, en particular las herramientas EBSCO (marca registrada, se refiere a una biblioteca digital de literatura académica existente en la colección de la Biblioteca de la USB) y Google. 
b. Desarrollar capacidades para detectar problemas susceptibles de ser desarrollados en el campo de la interacción entre tecnologías digitales de información y comunicación, y la participación estudiantil en la universidad venezolana.

Apoyados por nuestra experiencia como participantes en el MOOC de Siemens y Downes (el segundo dictado por ellos en Canadá, transcurría el año 2009) decidimos convertir nuestro curso en una experiencia CAMEL. Así, con el apoyo de la entonces Jefe de mi Departamento de adscripción en la Universidad se dictó un curso regular pero que podía ser tomado por otras personas y en formato virtual (recuerdo que muchos alumnos iban a mi oficina para ver en las computadoras la clase que me encontraba dictando en el salón vecino).

Entre los cursantes de esta materia electiva se encontraban cursantes de programas tan variados como ingeniería electrónica e informática, entre otras, y por ello dudaba del éxito del curso entre jóvenes para los que la informática y la computación era natural (en ese entonces creía en la brecha digital por razones etarias). Sin embargo, a lo largo del desarrollo del CAMEL fue tal la acogida por parte de los estudiantes, que tomé la decisión de crear una Fundación que permitiese el concebir, desarrollar e implantar programas que aseguren, tanto a individuos como a comunidades, el acceso y manejo eficiente de los recursos informáticos disponibles en Internet y el ser compartidos a través de las redes sociales. InterConectados nace, como organización sin fines de lucro, para ayudar a generar, promover y fortalecer la cultura participativa y colaborativa que debería caracterizar a esta nueva sociedad del conocimiento.

Para legalizar lo que creíamos en ese momento sería una ONG en posibilidad de dictar cursos y recibir recursos por sus actividades, generamos una directiva de tres personas, con la particularidad que una de ellas era uno de los alumnos que había tomado el curso de Impacto Social de Internet, una manera de incorporar personas jóvenes con nuevas visiones del mundo en el que deberían desenvolverse.

Una de las primeras actividades que realizamos fue la de crear el Blog de Interconectados, en el cual todavía llegamos a subir una nota, con fecha 5 de diciembre de ese año de 2011, socializando una de las últimas clases del curso de pregrado ya 
mencionado (Educación y TIC's: el papel de los docentes, 5-12-2011). Nuestra esperanza era que el Blog nos facilitase la difusión y el contacto con personas de intereses semejantes.

\section{Esfuerzos para desarrollar la Investigación Colaborativa}

La primera entrada en el blog de la Fundación remonta al 21 de octubre de 2011, una nota de saludo a los (futuros) lectores. Para el día siguiente ya estamos comentando sobre temas técnicos, una nota promocional que se tituló Herramientas de Aprendizaje "APA" que para entonces lo interpretamos como Ambientes para Aprender. Para el día de hoy, al meter en el buscador del blog de Interconectados ese acrónimo aparecen más de dieciocho entradas.

No obstante, estamos conscientes de los retos que enfrentamos en aquel entonces, y todavía: por una parte el bajo nivel de experticia en informática educativa, por no hablar de estrategias conectivistas, en la sociedad en general (Castillo y Ordóñez 2009) y entre estudiantes y docentes en nuestro país en particular (Ordóñez, 2009; García y Ordóñez, 2012)), quizás debido a la ausencia de una inserción transversal de la conectividad en los curricula de estudios generales universitarios (Ordóñez, 2011), lo que impide a los futuros docentes, en el caso de los cursantes de carreras en educación, dominar técnicas que luego no podrán profundizar a la hora de requerirlas para utilizarlas en la educación de sus estudiantes a todos los niveles. De igual modo la ausencia de este tema en las inducciones que hacen a sus profesores noveles las distintas carreras universitarias.

Por otra parte, los problemas de origen cultural que afectan nuestra manera de lidiar con la utilización de información para la toma de decisiones (Ordóñez, Adrián, Goncalves, 2013; Ordóñez, 2012) y que en el fondo también van a contribuir a dificultar la diseminación de las ya no tan nuevas tecnologías al evitar los docentes tener que aceptar que no las dominan como se esperaría de sus figuras de autoridad.

Muy rápidamente abordamos mediante el blog de Interconectados lo que era la preocupación inicial, divulgar las herramientas informáticas y explorar en la entonces naciente experiencia de los CAMEL. Así, en rápida sucesión, se dictaron entre el año 2011 y el año 2012 tres CAMEL, el primero sobre el tema Participación y Democracia desarrollado 
a lo largo de tres etapas; la primera y la segunda fueron cubiertas durante el año 2011. La tercera, desarrollada a lo largo del primer trimestre del 2012, devino en un experimento de alianza estratégica con el Doctorado Interdisciplinario en Ciencias Sociales y Humanidades de la Universidad Simón Bolívar en Caracas, Venezuela, donde formó parte de las actividades del Seminario en Participación que se dicta en ese programa. Así, se aspiró integrar en el CAMEL a los candidatos doctorales de la USB con el público interesado en el tema, a objeto de estimular la generación de redes y la aparición de Ambientes Personales de Aprendizaje (APA) por parte de todos los participantes y sus respectivos entornos. En nuestra visión era un CAMEL orientado al público general pero apoyado en un núcleo "duro" de doctorandos de alto nivel. Todos los materiales de ese CAMEL pueden aún encontrarse en la web desde nuestro blog.

El segundo CAMEL, dictado entre abril y julio del 2012, orientado a estudiantes universitarios y artesanos populares, que llevaba por nombre Emprendimientos Cooperativos en Conectividad, pretendía superar la visión “heroica” del emprendimiento, y pasar a una visión racional-moderna de lo que significa pensar en una empresa competitiva dentro de un entorno complejo. Este CAMEL tuvo la particularidad que se apoyó en una serie de destacados profesores y se realizó sin ningún tipo de apoyo formal de alguna institución académica. Todos los materiales utilizados se pueden bajar de la web desde nuestro blog.

Y es así como llegamos al tercer CAMEL de Interconectados, el cual se apoyó en la experiencia acumulada y nos permitió intentar esfuerzos colaborativos entre los participantes. El CAMEL Investigaciones Colaborativas en Ciencias Sociales fue dictado entre los meses de septiembre y diciembre del 2012, a lo largo de 12 semanas, en alianza estratégica con el Doctorado en Ciencias Sociales y Humanidades de la Universidad Simón Bolívar de Caracas, Venezuela(DSCH-USB). El mismo pretendía explorar el tema a lo largo de tres orientaciones fundamentales:

- Investigaciones Colaborativas en las Ciencias Sociales en el Mundo

- Investigación Colaborativa en Ciencias Sociales en América Latina 
- Investigación colaborativa entre diferentes programas doctorales en nuestro medio.

con el objetivo explícito que los participantes: "conozcan, comprendan y adelanten estudios colaborativos en su área de interés dentro de las ciencias sociales, bajo una perspectiva interdisciplinaria". Como siempre en Interconectados, el CAMEL estuvo abierto a todos los interesados, sin distingos de carrera o grado académico alcanzado; y totalmente gratuito. Los materiales utilizados para este CAMEL también pueden ser obtenidos en la web.

A partir de este CAMEL, y quizás por la particularidad de haber contado entre sus participantes con docentes universitarios provenientes de múltiples instituciones y con diferentes grados de desarrollo académico, comenzamos a comprender que en el fondo lo que perseguimos y debemos profundizar aún más es ahondar en el problema de cómo movilizar conocimiento entre la universidad y la sociedad. Cómo lograr que individuos inteligentes e interesados lograsen por la vía de la colaboración, integrar experiencias y hallazgos científicos y aplicarlos a la solución de problemas en su entorno. Pareciera que esta nueva variable o punto de vista nos lleva entonces a preocuparnos por nuevos aspectos tales como la complejidad de las relaciones entre instancias al interior de la universidad, o entre la universidad y la sociedad, al igual que la necesidad emergente de atender a los problemas de gerencia de grupos de investigación y la díada conformada por la interacción profesor-estudiante a la hora de adelantar tareas como los servicios comunitarios, obligatorios en la universidad venezolana.

Nos encontrábamos entonces frente a los CAMEL de movilización de conocimientos.

\section{La movilización de conocimientos entre Universidad y Sociedad}

Para este momento, agosto de 2015, el buscador del blog de Interconectados detecta 43 entradas sobre investigación colaborativa y 39 entradas sobre movilización de conocimientos entre universidad y sociedad (algunas de las entradas aparecen en ambas búsquedas). 
Los datos anteriores nos hablan de la importancia que le damos a ambos temas, y que podemos resumir en nuestra creencia de que sin investigaciones colaborativas no podremos lograr movilización de conocimientos entre la universidad y la sociedad.

El esfuerzo de Interconectados por apoyar la movilización de conocimiento entre universidad y sociedad ha avanzado por dos caminos; por una parte los CAMEL sobre el tema, dictados posteriormente al de Investigaciones Colaborativas:

- CAMEL Organizaciones con Pertinencia Social (2014)

- CAMEL Taller sobre Estructuración de Problemas Complejos (20142015)

- que además de los CAMEL Cursos sobre Conectividad para Educadores, dictados colaborativamente con AsoVAC (la Asociación Venezolana para el Avance de la Ciencia), constituyen el grueso de nuestra labor.

Adicionalmente, hemos incursionado en la organización de foros sobre el tema de la movilización de conocimientos entre la universidad y la sociedad en el marco de la Convención Anual de la AsoVAC, retomaremos este último punto más adelante.

¿Cómo se organizan en la actualidad los CAMEL sobre Movilización de conocimientos entre la universidad y la sociedad?

Estructura: el seminario se concretiza en tres bloques temáticos:

1. Herramientas de informática educativa para la acumulación y socialización de información

2. Investigación sobre "los trabajos de grado y la movilización de conocimiento en la sociedad latinoamericana"

3. Estrategias para la investigación colaborativa en Ciencias Sociales

Objetivo General: Se pretende que el participante conozca, comprenda y diseñe investigaciones colaborativas en su área de interés bajo una perspectiva interdisciplinaria utilizando herramientas de informática educativa.

Los objetivos específicos incluyen: 
1. Comprender, analizar y sintetizar la importancia de los paradigmas y las culturas organizacionales en el quehacer universitario, en particular en lo referente a la generación y movilización de conocimiento dentro de la propia universidad y con su entorno social..

2. Apoyar la estructuración del problema a tratar en la Tesis Doctoral de los participantes, o en los temas de investigación individuales, en función de las demandas societales.

3. Formular un proyecto de investigación interdisciplinario colaborativo en el ámbito de interés de su tesis doctoral o intereses de investigación.

Como parte de la evaluación de lo logrado en los CAMEL, en estos momentos cada participante en una primera fase, o grupos de participantes (tres o cuatro) en la fase final, deben realizar una presentación, la cual debe subirse a la plataforma YouTube en el formado de cinco láminas máximo, para un tiempo no mayor de 10 minutos. En relación a nuestra propia evaluación de lo logrado hasta ahora pensamos que el énfasis debe ser puesto en el número de participantes y en los avances logrados en relación a la cultura colaborativa que perseguimos. Ciertamente nuestros CAMEL no son masivos en el sentido de la cantidad de participantes, dos a tres decenas es el número usual que se registran para los mismos. Aunque aceptamos que nuestro enfoque de masivo se refiere mas a que los participantes provengan de múltiples instituciones y países, con diferentes niveles de formación y títulos profesionales debido al hecho de ser desarrollos orientados a personas con nivel de postgrado. En relación a la culminación, lo usual es que los participantes que asisten a los CAMEL como parte de una materia regular de sus programas de postgrado los culminen llevando adelante las asignaciones, mientras que los demás participantes que culminan lo hacen como observadores. Temas que quedan para futuras exploraciones por expertos en educación a distancia y evaluación de estrategias pedagógicas. Por nuestra parte, la experiencia obtenida y la oportunidad de incorporar estas experiencias en desarrollos sucesivos ha sido el principal acicate para adelantar estos esfuerzos.

Con relación a los Foros sobre movilización de conocimientos, hasta la fecha se han realizado dos, en 2013 y 2014 respectivamente. El primero fue un esfuerzo por analizar las diferentes estructuras universitarias que podrían tener relación con el objetivo 
de movilizar conocimiento a la sociedad. Para ello presentamos el evento como un esfuerzo de recopilación de posibilidades, en el sentido de que la universidad es un elemento clave en la movilización de conocimiento en una sociedad. No solo por la información que maneja sobre los más diversos campos y disciplinas (la base del conocimiento en ingeniería, medicina, leyes y demás) sino por su capacidad de generar nuevo conocimiento gracias a la investigación y por su capacidad de movilizar este y aplicarlo gracias a la docencia y a la extensión. La universidad bien utilizada es una herramienta fundamental en la lucha de las sociedades por alcanzar cada día más y mejores niveles en su calidad de vida.

Pero al igual que una fábrica es algo más que una línea de producción, una universidad es algo más que una acumulación de docentes impartiendo clases. La información presente en sus bibliotecas, los espacios de interacción que pueden establecer sus servicios de prensa y comunicación social, así como la vasta red de vasos comunicantes en que se constituyen sus egresados, forman también parte del enorme y complejo reto que se le presenta a la gerencia universitaria a la hora de alinear sus diferentes componentes para ponerlos al servicio de la sociedad, indistintamente de su “habitus" (Bourdeau), o que por su propia naturaleza sea una "organización débilmente acoplada" como ha sido definida (Ingersoll, a993; Weick, 1976). Todo esto fue evaluado en el desarrollo de Foro y las láminas utilizadas en las presentaciones pueden ser accesadas desde nuestro blog.

El segundo Foro se inicia con un reconocimiento al Dr. Luis Cortes, bajo cuyo liderazgo como Decano se produjo el traslado de la Facultad de Ciencias de la Universidad Central de Venezuela (UCV), desde una serie de espacios dispersos hasta su actual sede, donde se realizaba el evento en cuestión. Igualmente, se recordó a las figuras de Francisco De Venanzi (papel protagónico en el surgimiento de AsoVAC y la Fac. de Ciencias de la UCV) y Arístides Bastidas (promotor del periodismo científico en Venezuela), ilustres ejemplos en nuestro país de lo que significa insertar la ciencia en una sociedad.

Las áreas a explorar por los ponentes invitados fueron: las estructuras organizativas universitarias para la prestación de servicios, las particularidades de los 
docentes y estudiantes involucrados en los procesos requeridos, y el impacto de las organizaciones extrauniversitarias en la interacción universidad-sociedad. Los objetivos específicos por área fueron, a título indicativo más no excluyente, los siguientes: Las estructuras organizativas universitarias: Cuáles son, cómo funcionan, cómo aprenden y cómo evalúan resultados las empresas y fundaciones universitarias responsables del apoyo a empresas y organismos públicos y privados en la sociedad. Las particularidades de los docentes y estudiantes: Factores que inhiben o estimulan la participación en procesos de movilización de conocimientos entre la universidad y la sociedad. Influencias familiares o institucionales, comunicación profesor-estudiante-comunidad, factores motivacionales. Impacto de las organizaciones extrauniversitarias en la interacción universidad sociedad: Los efectos "push-pull” en la interacción universidad-empresa. Problemas de comunicación entre los generadores de conocimiento y los usuarios de conocimiento. Al igual que en el primer Foro y en nuestros CAMEL, las ponencias presentadas pueden ser obtenidas directamente en las páginas del blog, con la particularidad de que en el caso del II Foro, totalmente virtual, en adición a las láminas presentadas se conservaron las ponencias con audio, subidas en la plataforma Youtube, de manera que se puede ver y oir cada una de las presentaciones visitando el enlace correspondiente.

En el momento de escribir esta recopilación avanzamos en los preparativos para celebrar el III Foro, en esta oportunidad totalmente dedicado al tema de los servicios comunitarios, actividad obligatoria por Ley (2005) para los estudiantes que aspiran a egresar de las universidades venezolanas, y con la aspiración plasmada en su artículo cuarto:

... se entiende por Servicio Comunitario, la actividad que deben desarrollar en las comunidades los estudiantes de educación superior que cursen estudios de formación profesional, aplicando los conocimientos científicos, técnicos, culturales, deportivos y humanísticos adquiridos durante su formación académica, en beneficio de la comunidad, para cooperar con su participación al cumplimiento de los fines del bienestar social, de acuerdo a lo establecido en la Constitución de la República Bolivariana de Venezuela y en esta Ley. 
De manera que nos acercamos gradualmente, al menos a nivel teórico. al logro de la meta de apoyar la movilización de conocimientos entre la universidad y la sociedad.

Pero a pesar de los CAMEL, de los Foros, de las múltiples iniciativas generadas, es poco lo que hemos avanzado en el objetivo de generar investigaciones colaborativas que faciliten la inserción de conocimiento en la toma de decisiones en nuestro medio. Algunas explicaciones en la próxima sección.

\section{Problemas en la colaboración tanto para generar como para movilizar conocimientos en nuestro médio}

Son múltiples los factores que deben ser analizados a la hora de tratar de entender cómo lograr una mayor cantidad de procesos colaborativos para la solución de problemas en nuestra sociedad. Muchos de los factores hasta aquí mencionados pueden ser visualizados en un diagrama similar al desarrollado por Olstrom (2007) en su artículo sobre cómo ir más allá de las panaceas en el manejo de problemas socio-ecológicos, basado en sistemas de problemas anidados sucesivamente, pero añadiendo las variables críticas para la gestión de conocimiento que asume Leistner (2010) a saber: la gente o capital humano, los procesos que responden a una estrategia, la cultura o capital social, y la tecnología, todo lo cual nos permite visualizar un marco de referencia para apreciar la dimensión del problema entre manos.

Figura 3 - Factores que afectan la colaboración y la movilización de conocimientos, después de Leistner (2010)y Ostrom (2007).

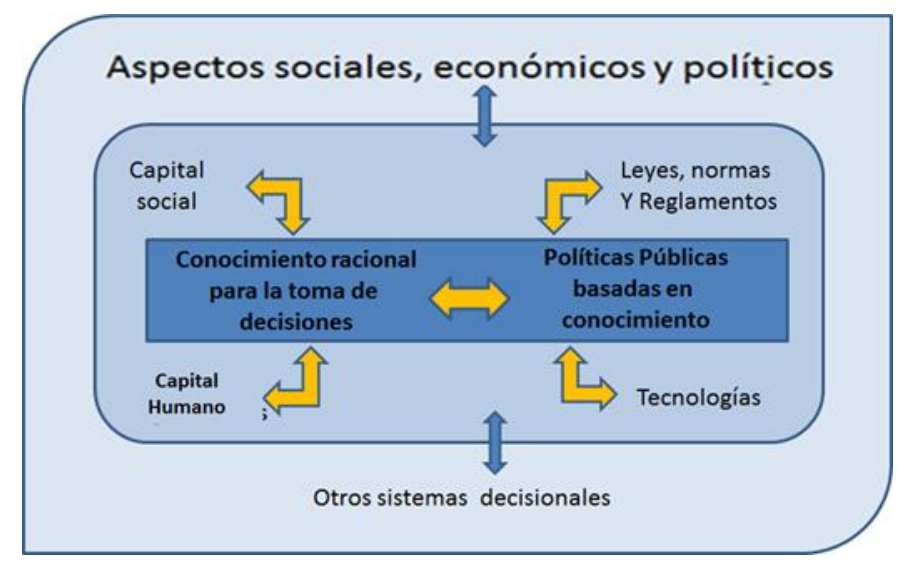

Elaboración propia. 
En lo que sigue trataremos de concentrarnos en los aspectos tratados hasta ahora por nosotros en las diferentes entradas de Interconectados.

\section{Desde el capital social}

Quizás el primer punto que deba analizarse al tratar de adentrarnos en los cuellos de botella que deberán resolverse antes de lograr investigaciones colaborativas que permitan una movilización de conocimientos eficiente entre nuestras universidades públicas y la sociedad está relacionado con la investigación sobre "Infancias desiguales" realizada por Lareau (2003) que demuestra como, dentro de la sociedad norteamericana, la raza, el género, la religión y la clase social, son sumamente importantes a la hora de definir logros en la vida. Mediante un estudio etnográfico de la vida de doce familias con niños en tercer grado de escolaridad, la autora descubre la lógica de crianza familiar que reproducirá las desigualdades en las que están inmersos los padres de esos niños, y que los afectará a lo largo de sus vidas.

El estudio de Lareau viene a cuenta al observar cómo, en una evaluación cualitativa de nuestras universidades venezolanas y sus bibliotecas, realizada con los cursantes regulares de nuestro Curso Abierto Masivo en Línea sobre Investigaciones Colaborativas en Ciencias Sociales, encontramos rasgos que parecieran mantener la cultura tradicional de nuestras organizaciones. Nos referimos a la visión de las universidades como "islas de conocimiento", separadas de otras estructuras sociales por paredes sumamente difíciles de penetrar, y que imponen a sus miembros visiones similares a las ya preexistentes, dificultando así el cambio necesario (y posible gracias a las tecnologías digitales).

Ya en el pasado se describió el concepto de insularidad en notas del blog de Interconectados (ver por ejemplo la nota sobre la isla del conocimiento). Lo utilizamos en el sentido exacto que le asigna Mario Waissbluth en su conocido artículo La insularidad en la gestión pública latinoamericana, así “... existe un fenómeno estructural y endémico: la “insularidad” o “feudalización” de la gestión, caracterizada porque en la gran mayoría de estas instituciones la queja más frecuente es que cada unidad, división, departamento o subsecretaría trabaja como una isla separada, y en el peor de los casos, con franco antagonismo, mala comunicación y traslapo de funciones entre ellas". Sentimos (no lo 
hemos demostrado) que en el terreno de los espacios de investigación de la universidad pública latinoamericana existe una situación similar. Esta situación afecta procesos deseables como los de la inserción transversal de conectividad en los estudios generales universitarios (Ordóñez, 2011) o el convertir a la universidad en cantera de liderazgos políticos (Pittari y Ordóñez, 2011) que requieren por definición interacción eficiente entre diferentes componentes de la organización universitaria.

“Relaciones desiguales" pudiera entonces llamarse el libro que resultase de un estudio a fondo sobre cómo nuestra sociedad utiliza información y conocimiento, dependiendo de si proviene de una institución democrática y meritocrática como la universitaria o si, por el contrario, proviene de la autoridad percibida o establecida, como puede ser el poder de los gobiernos, sean estos locales, regionales o nacionales. De allí la importancia de aprender a movilizar información proveniente de la investigación científica a los lugares de toma de decisión de políticas públicas. Para ello las universidades deben comenzar por abrirse a la interacción con las comunidades que las rodean, conocer lo que estas necesitan y practicar a proveer ese conocimiento necesario. Solo con esta experticia podrá la universidad intentar el salto cuántico de intervenir en las decisiones de política de manera orgánica de forma sistemática.

Las entradas de interconectados que se refieren a este tema son variadas y tratan fundamentalmente sobre las características culturales propias de nuestro medio. Si reconocemos que en América Latina contamos con una cultura propia que nos diferencia de las culturas de otros pueblos del mundo y que ella a su vez incide en los usos y costumbres con que enfrentamos el quehacer ciudadano en nuestros entornos, nos encontramos que los mayores retos a vencer parecieran estar presentes en las áreas del desarrollo de culturas computacionales y colaborativas en nuestros académicos, para la comunicación y conformación de equipos eficientes entre ellos y con la sociedad que los circunda. Esta debe ser la meta inmediata en los programas de postgrado de nuestras universidades: ayudar mediante estrategias didácticas adecuadas a facilitar el desarrollo de las competencias requeridas por los nuevos docentes - investigadores universitarios requeridos en la sociedad del conocimiento. 
Si a lo anterior añadimos el problema de no saber airear nuestras diferencias, de no lograr discutir sobre ideas y conceptos sin por ello personalizar los procesos de discusión, de manera que podamos seguir trabajando colaborativamente luego de resolver las visiones encontradas que pudieran surgir en el proceso de interactuar, tenemos que concluir que para la universidad venezolana, y por extensión nos atrevemos a incluir la de toda latinoamérica, va a resultar muy difícil abrirse a interacciones abiertas con la sociedad que la rodea. Esto también dificulta entre nosotros el manejar los “comportamiento oportunistas" producidos cuando una parte toma ventaja de su superior conocimiento, a fin de lograr mayores resultados al no divulgar dicha información a la otra parte. Este fenómeno es frecuente en nuestro medio, entre otras razones por las culturales ya comentadas.

\section{Desde el punto de vista de las Leyes, Normas y Reglamentos}

Ya nos hemos referido en el pasado a como las normas y reglamentos para los estudios de postgrado en nuestro país no contribuyen a fomentar culturas o procesos de investigación colaborativos (Ordóñez, 2015b), pero no es solo a este nivel que se encuentran los obstáculos. El colaborar entre seres humanos es siempre difícil. Trátese de investigaciones para generar nuevos conocimientos, o de emprendimientos para aplicar conocimientos en forma novedosa, el reunir personas de diferente formación, con diferentes historias y hasta diferentes culturas en un equipo eficiente es una tarea cuesta arriba, pero siempre susceptible de ser analizada y facilitada por la reflexión crítica.

Un ejemplo concreto lo encontramos en la Comisión Suiza para Investigaciones Colaborativas con Países en Desarrollo que publicó una breve Guía para Investigaciones Colaborativas Transfronteras (2014) que consta de once principios y siete preguntas clave para ayudar a colaborar en una amplia gama de situaciones, desde investigadores que intentan asociarse para proyectos coordinados, hasta organizaciones internacionales que pretenden resolver con conocimiento los grandes problemas que enfrenta la humanidad.

Los once principios son:

1. Fijar la Agenda de Trabajo en forma conjunta

2. Interactuar con todos los públicos involucrados 
3. Aclarar y definir las responsabilidades

4. Rendir cuentas a los beneficiarios

5. Promover el aprendizaje mutuo

6. Incrementar las capacidades de todos los participantes

7. Compartir datos y redes

8. Divulgar los resultados

9. Cosechar juntos los honores y los beneficios

10. Aplicar los resultados

11. Asegure la continuidad de los beneficios que resulten

Y las siete preguntas que deben hacerse en forma permanente los participantes en proyectos colaborativos son:

1. ¿Por qué trabajamos en forma colaborativa?

2. ¿Cómo aseguramos la cohesión del equipo?

3. ¿Cual forma de colaboración debemos utilizar?

4. ¿En qué nos enfocamos y cuales son las prioridades?

5. ¿A quienes más involucramos?

6. ¿Donde creamos relevancia?

7. ¿Cuando consolidamos resultados?

En este sentido Bammer (2006) ha señalado que no existe una sola manera de describir o explicar lo que significa el término "integración" en el contexto de la investigación, por lo que los recuentos disponibles varían mucho en contenido y énfasis; de ahí que propone un marco de trabajo basado en varias preguntas orientadoras:

1. ¿Qué pretende lograr la integración y quiénes se benefician de ella?

2. ¿Qué se va a integrar?

3. ¿Quién integra?

4. ¿ Cómo se lleva a cabo la integración?

$5 \cdot$ ¿ Cual es el contexto de la integración?

6. ¿Cuál es el resultado de la integración?

El peor enemigo de este proceso de intentar realizar esfuerzos colaborativos es lo que los angloparlantes llaman "wishful thinking" (traduce algo así como "hacerse ilusiones" o "buenos deseos") y que preferimos llamar en nuestro ámbito 
latinoamericano "macondismo", (Volek, 2007), el creer que por imaginarnos algo ya lo hemos logrado. Nos topamos en forma permanente con "proyectos de investigación" que se limitan a describir una situación problemática y saltan a concluir que "entonces lo resolvemos", sin estructurar el problema en cuestión, reflexionar sobre los procesos administrativos involucrados o definir los protocolos de investigaciones de campo propiamente dicha. Contra todo esto deberá luchar en su esfera personal cada investigador universitario antes de salir al mundo a tratar de generar, o incorporarse a, redes de investigadores interesados en colaborar para solucionar los problemas que presenta su entorno social. Y para ello su principal aliado será el conectivismo.

Lo anterior en parte explica las quejas sobre las investigaciones que se realizan en las universidades y el hecho de que no ayudan a resolver los problemas de las sociedades donde se encuentran inmersas. Independiente de que no todas las investigaciones académicas tienen que estar necesariamente atadas a la resolución de un problema social, lo cierto es que, al menos en parte, la razón de encontrar tan pocos casos de investigaciones exitosas con relación a problemas sociales pasa por la dificultad de concebir, organizar y adelantar los esfuerzos colaborativos que requieren, por lo general, la investigación y resolución de los problemas puntuales de una sociedad. Tratamos de visualizar el proceso en el siguiente mapa conceptual:

Figura 4.- Estrategias para la búsqueda de colaboradores para proyectos de investigación. (Elaboración propia)

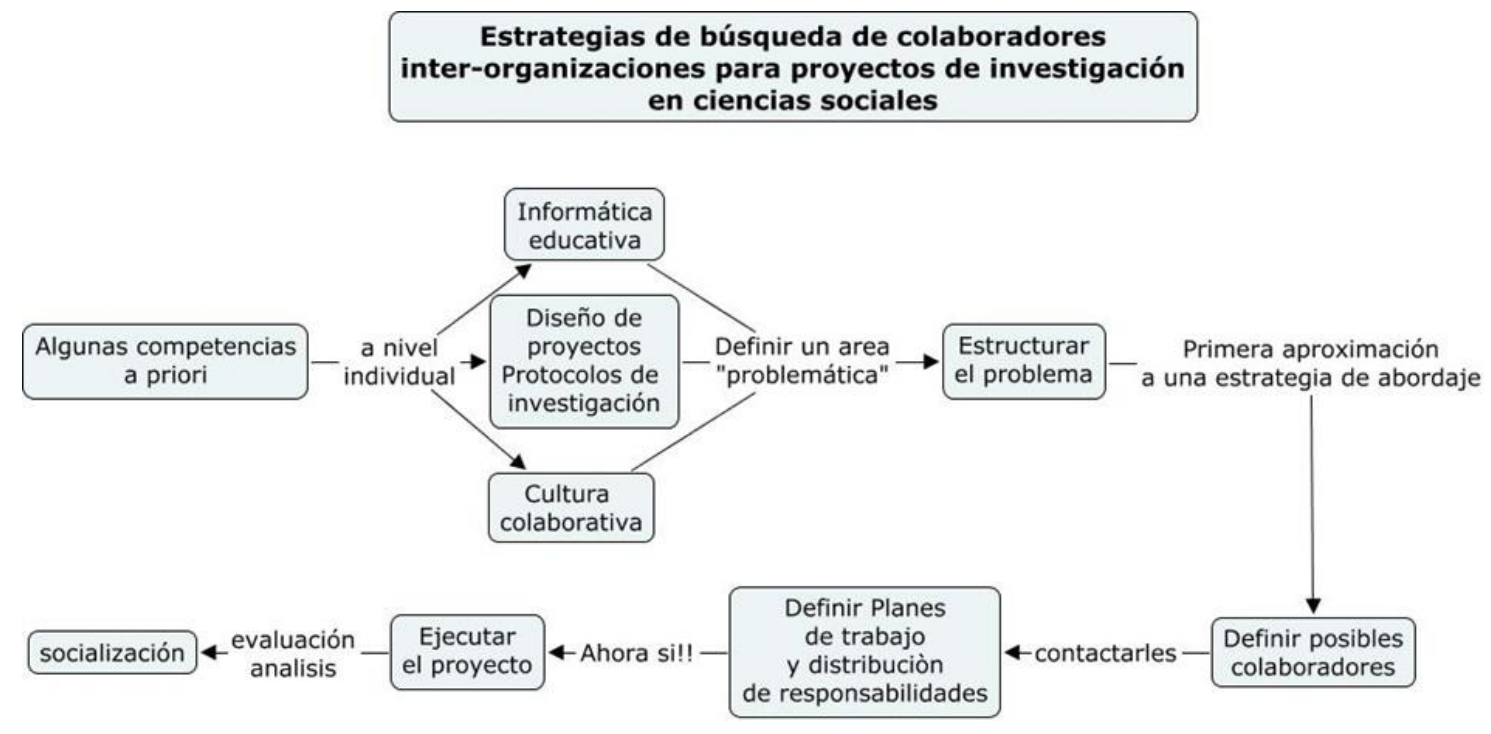


Podemos así intentar visualizar una aproximación al análisis del problema. En primer lugar, debemos considerar las competencias que deben poseer los investigadores involucrados. Por lo general no es alta la experticia del profesor universitario de nuestro medio en tópicos tales como informática educativa, diseño de protocolos de investigación y las ya analizadas: cultura colaborativa y estrategias sociales para la colaboración. Si bien es cierto que podemos desarrollar en lapsos relativamente breves competencias instrumentales en informática y generación de protocolos, ya hemos abundado sobre el hecho de que la cultura colaborativa choca en mucho con la cultura prevalente en nuestra sociedad autoritaria y motivada al poder, por lo que una aproximación a la solución de este cuello de botella debe pasar por racionalizar el problema y buscar a nivel individual estrategias de solución. Esto último deberá ser un esfuerzo permanente en todos los procesos y no será suficiente, por enfrentarse a las realidades sociales existentes en los entornos que rodean al investigador, tanto en su medio académico como en la sociedad en general.

Pero en adición a las competencias individuales necesarias, el investigador interesado en realizar investigaciones sociales colaborativas también debe disponer del tiempo necesario para estructurar adecuadamente el problema en cuestión. Para ello acudimos a la teoría generada para el análisis de políticas públicas, de acuerdo a William N. Dunn (2014), que nos dice que debemos pasar de la sensación de problemas a los problemas de política, mediante su estructuración, que en cierta forma se refiere a descomponer lo que se desea estudiar en sus partes más importantes, conocer a fondo la literatura pertinente que le permita detectar otros posibles investigadores académicos interesados en el tema y negociar con estos los planes de trabajo, distribución de responsabilidades y demás aspectos administrativos de todo proyecto, por no hablar del tiempo requerido para detectar, convencer y generar redes de colaboración entre los investigadores.

Aún más, antes de realizar los proyectos de investigación con impacto social, él o los investigadores deberán invertir tiempo y esfuerzos adicionales para generar una estructura social de colaboración en red con autoridades, comunidades, actores importantes y demás, quienes de una u otra manera están involucrados en el desarrollo 
exitoso de un proyecto de intervención social, o de investigación que lleve a esa posible intervención en aras del mejoramiento de la calidad de vida de una población. Tan solo uno de los aspectos a considerar es el de la inadecuación de la organización universitaria para el apoyo de emprendimientos colaborativos de profesores individuales con la sociedad, abocada como está la mayor parte de la estructura a las labores de docencia. Como ejemplo, el ya mencionado caso de las bibliotecas universitarias (Ordóñez et al, 2013)

En la medida que se profundiza el "habitus" universitario, generado dentro del paradigma de la universidad generadora de conocimiento, que incentiva la publicación académica como un fin en sí mismo, se produce una situación en que la mayor motivación para publicar tiene que ver con las luchas propias de la violencia simbólica en el campo universitario. Así, a mayor número de publicaciones mayor posibilidad de ascenso académico lo cual, unido a los estímulos de tipo económico como las "carreras de investigador", conducen a una búsqueda permanente de este tipo de metas por parte de los miembros de la academia. El capital cultural así generado va a ser utilizado posteriormente para diferenciarse de aquellos que no publican, no solo los individuos que están fuera del campo universitario, sino los que en el interior de él no participan de la carrera académica, como son los profesionales a cargo de las labores administrativas. Lo anterior tiene varias consecuencias, la menor de las cuales no es que, en busca del mínimo esfuerzo (los amigos economistas podrían profundizar sobre este punto), el investigador va a tender a investigar en aquello que ya conoce, y a la hora de colaborar con otros investigadores lo hará para reunir lo que ya ambos dominan y donde les resulta más fácil publicar. En el camino, y debido a los sistemas de incentivos y del mínimo esfuerzo, generados al haber convertido la publicación en un fin en sí misma debido al habitus académico, las investigaciones dejan de orientarse a resolver las necesidades de la sociedad y se tornan parte de los rituales preestablecidos por el mismo mundo académico.

La situación descrita tiene importantes consecuencias a la hora de orientar nuestra atención al problema de la movilización de conocimientos entre la universidad y la sociedad. El sistema de variables (normas y reglamentos, ¿valores?) vigente orienta las investigaciones hacia el interior de la organización universitaria. De esta forma 
difícilmente podrá el conocimiento generado por esas investigaciones ser de interés para la sociedad.

\section{A modo de conclusiones y recomendaciones}

Si aceptamos que investigar los problemas sociales que actualmente enfrentamos representa entender problemas complejos de difícil solución, podemos entender la dimensión del reto que se le presenta a los investigadores científicos y a la universidad como estructura organizativa a la hora de acometer la generación, movilización y aplicación de conocimientos en estas áreas problemáticas. De manera que el primer obstáculo a vencer por los investigadores interesados en generar conocimiento para la sociedad es el de definir problemas de esa realidad, para luego conformar los equipos interdisciplinarios que garanticen que los problemas serán analizados desde diferentes ángulos, a objeto de lograr claras visiones producto de esta mirada holística, bastante diferente de muchas de las investigaciones en curso en nuestras universidades en la actualidad. Y para todo ello es indispensable contar con herramientas adecuadas para la información y la comunicación en los volúmenes y variedad requeridas. Ya no basta con leer y escribir, como en una época llegó un momento en que no bastaba con hablar, ahora debemos poder utilizar las tecnologías que nos permiten explorar amplios volúmenes de información, organizarlos y presentarlos a otros colaboradores con la esperanza de encontrar los patrones y leyes que rigen los hechos bajo observación.

Por otra parte, la organización universitaria debe transformarse, desde su presente paradigma de educación, investigación y extensión a otro basado en la movilización y generación del conocimiento requerido para resolver los problemas de la sociedad. La sociedad como usuaria de conocimiento debe estar en el centro de las preocupaciones de la organización universitaria, solo así generará los mecanismos requeridos para apoyar a sus integrantes (docentes, funcionarios y estudiantes) en las tareas propias del quehacer social que le debe ser consustancial. Para este proceso es fundamental que la comunicación entre la universidad y la sociedad sea una "autopista de dos vías", de manera que cualquier comunidad o instancia en esa sociedad pueda 
expresar sus necesidades con facilidad y obtener respuestas adecuadas sin importar lo complejos que puedan ser los procesos al interior de la universidad. Una imagen adecuada nos la brinda la misma internet, con las plataformas de venta en línea, donde usted dice lo que quiere y el proveedor lo envía a su domicilio sin que usted tenga que preocuparse de dónde lo obtuvo el proveedor y qué tuvo que hacer para garantizar que usted lo recibiera.

Para concluir, debemos referirnos entonces al problema propio de la colaboración entre la universidad y los decisores de políticas públicas. En el esquema lineal al que nos referíamos en la figura $\mathrm{N}^{\circ} 1$, esta labor se deja, en cada caso, al docente-investigador experto en un aspecto determinado del conocimiento. Nada más ineficiente y frustrante, Ya hemos comentado en múltiples oportunidades en el blog de Interconectados el viejo dilema sociológico de lo que debe saber el científico y lo que debe saber el político acerca de esa relación. Este papel de comunicar a los dos sectores debe ser asumido desde la universidad por la organización universitaria, que no por los docentes. Pero para ello requiere profundizar en sus experiencias colaborativas al interior de la organización, entre sus diferentes elementos, y al exterior con la sociedad.

Algunos de estos temas han sido ya tocados por nosotros en charlas o conferencias que se encuentran disponibles en la web desde nuestro blog, la más recientes con motivo de estar celebrando 40 años de creada la revista Ciencia y Sociedad, órgano de divulgación científica del Instituto Tecnológico de Santo Domingo, cuando se dictó una conferencia sobre el tema de la movilización de conocimientos, que nos permitió poner en el tapete algunos temas relacionados con la crisis de la educación superior, desde aspectos claves de la gestión universitaria hasta aquellos relativos a las diversas expresiones de la comunicación científica en latinoamérica. La conferencia puede ser accesada en la web: En forma similar, una charla analizando este problema fue recientemente presentada en el marco del Primer simposio de intercambio binacional universitario que tuvo lugar en la Facultad de Ciencias Económicas y Sociales de la Universidad de Carabobo en Venezuela. Se puede accesar a la charla desde aquí. En ambos casos intentamos analizar el problema desde la visión que nos brinda el estudio de sistemas complejos. 
Aunque las teorías sobre gestión de sistemas complejos vienen siendo estudiadas desde hace algún tiempo, es solo recientemente que el problema de cómo manejar sistemas complejos en ciencias ha venido siendo abordado. Uno de los intentos más clarificadores, en nuestra opinión, es el de las ciencias de integración y aplicación de conocimientos, desarrollado por Gabriele Bammer (2013) en la Universidad Nacional de Australia y conocido coloquialmente por sus siglas en inglés: I2S (derivado del inglés Integration and Implementation Sciences). Pero estos estudios aún aguardan por ser realizados en nuestro medio latinoamericano. Quizás la solución en el nivel operativo se encuentre en la utilización de caminos más estructurados para generar procesos colaborativos como los modelos $12 \mathrm{~S}$, pero eso será cosa del futuro, en primer lugar debemos tener conciencia de los problemas hasta aquí detectados y tomar la decisión de enfrentarlos si es que queremos mejorar el impacto de la universidad pública latinoamericana en la sociedad que la acoge y a la que se debe.

Mientras tanto, ¿cuál sería el valor estratégico de InterConectados en estos procesos que identificamos como necesarios para lograr las evoluciones positivas que pretendemos para la universidad pública latinoamericana? Por una parte consideramos conveniente mantener su papel como promotora de las TIC en nuestro medio, que aún se encuentra recorriendo la curva de aprendizaje requerido para su total incorporación en nuestras sociedades (facilitando la adquisición de una cultura computacional). Por otra parte, discutiendo y facilitando la generación de los intercambios de conocimientos requeridos para comprender racionalmente los procesos sociales en nuestro medio (facilitando la adquisición de una cultura colaborativa) para eventualmente tratar de convertirse en plataforma o lugar de encuentro entre generadores, movilizadores y usuarios de conocimiento en la meta común de generar políticas públicas basadas en la racionalidad que permitan ir resolviendo los problemas que se encuentran en nuestras sociedades. Seguiremos intentando utilizar Interconectados y su blog como herramienta para divulgar estos temas, y facilitar la incorporación de las tecnologías digitales en el ámbito latinoamericano. 


\section{Referencias}

AGUILLO, Isidro; GRANADILLO, Begoña \& LLAMAS, Germán., Posicionamiento en el Web del sector académico iberoamericano. Interciencia, 30. (2005)

BAMMER, Gabriele. (2013). Disciplining Interdisciplinarity: Integration and Implementation Sciences for Researching Complex Real-World Problems. ANU E-Press, Canberra.

CASTILLO, Ricardo \& ORDÓÑEZ, Luis. Los partidos políticos venezolanos y su aproximación a internet: una mirada no tecnológica. Temas de Comunicación, 18. (2009)

CETTO, Ana \& VESSURI, Hebe. LATIN AMERICA AND THE SPANISH-SPEAKING CARIBBEAN. UNESCO Science Report 2005 (pág. 45-73). París: Unesco (2005).

DUNN, William. (2011). Public Policy Analysis. Estados Unidos.

García, Gustavo \& ORDÓÑEZ, Luis. Internet y la participación ciudadana juvenil: una página en construcción. REIRE, 5. (2012)

LEISTNER, Frank. Mastering organizational knowledge flow: how to make knowledge sharing work. Wiley and SAS, Estados Unidos (2010)

OSTROM, Elinor. (2007). A diagnostic approach for going beyond panaceas. PNAS, Estados Unidos.

ORDOÑEZ, LUiS. INFORMACIÓN Y TECNOLOGÍA: EL CASO DEL MOVIMIENTO ESTUDIANTIL VENEZOLANO. IV Congreso de CiberSociedad. España: Observatorio para la Cibersociedad (2009). Disponible en:

http://www.cibersociedad.net/congres2009/es/coms/informacion-y-tecnologia-el-casodel-movimiento-estudiantil-venezolano/289/. Accedido el 13 de octubre de 2015.

ORDOÑEZ, Luis. La inserción transversal de la conectividad en el currículum de los estudios generales. Ciencia y Sociedad, XXXVI, (2011)

ORDOÑEZ, Luis, Cultura participativa y conectivismo: algunos retos para la investigación latinoamericana. Diálogos y desafíos euro-latinoamericanos: Ensayos sobre cooperación, derecho, educación y comunicación (pág. 289-308). Ed. Universidad del Norte, Barranquilla. (2012).

ORDOÑEZ, Luis; ADRIAN, Sixta \& GONCALVES, Nolberto. Información o Comunicación: El falso dilema de las bibliotecas Universitarias. ARJÉ Revista de Postgrado FACE-UC, 12. (2013)

ORDOÑEZ, Luis. La universidad y la movilización de conocimientos en la sociedad: el caso venezolano. Ciencia y Sociedad, 40(2). (2015a) 
ORDOÑEZ, Luis. La movilización de conocimientos en la sociedad: un reto para los programas doctorales en la universidad pública latinoamericana. Ciencia y Sociedad, 40(4). (2015b)

PITTARI, Enzo \& ORDOÑEZ, Luis. Un reto para los estudios generales: El Movimiento Estudiantil Latino Americano (MELA) como cantera de líderes políticos para el continente.

UMBRAL, 4. (2011)

SADOWSKY, George. Accelerating Development Using the Web: Empowering Poor and Marginalized Populations George Sadowsky. World Wide Web Foundation, Estados Unidos. (s/f).

SANCHO, Rosa; MORILLO; Fernanda; DE FILIPPO, Daniela; GÓMEZ, Isabel; FERNÁNDEZ, María. Indicadores de colaboración científica inter-centros en los países de América Latina. Interciencia, 31(4). (2006).

SIEMENS, George. Connectivism: A learning theory for the digital age. (2014). Disponible en: http://er.dut.ac.za/handle/123456789/69. Accedido en: el 13 de octubre de 2015.

STÖCKLI, Bruno; URS, Jon-Andri. A Guide for Transboundary Research Partnerships: 11 Principles. Swiss Commission for Research Partnerships with Developing Countries (KFPE), Suiza. (2014). Disponible en: http://www.naturalsciences.ch/organisations/kfpe. Accedido en: el 30 de agosto del 2015.

VOLEK, Emil. Anverso y reverso del laberinto de la soledad: Octavio Paz y cien años de Macondo. Cuadernos del CILHA, 8(9). (2007)

WAISSBLUTH, Mario. La insularidad en la gestión pública latinoamericana. Revista del CLAD Reforma y Democracia, 27. (2003). 